\title{
Critical appraisal of trastuzumab in treatment of advanced stomach cancer
}

\author{
This article was published in the following Dove Press journal: \\ Cancer Management and Research \\ 3 March 201I \\ Number of times this article has been viewed
}

\author{
Judith Meza-Junco \\ Heather-Jane Au \\ Michael B Sawyer \\ Department of Oncology, \\ Cross Cancer Institute, Edmonton, \\ Alberta, Canada
}

Correspondence: Judith Meza-Junco Department of Oncology, University of Alberta Cross Cancer Institute, I I 560 University Avenue, Edmonton, Alberta, Canada T6G IZ2

$\mathrm{Tel}+\mathrm{I} 7805778125$

Fax +I 7804328888

Email judith.meza-junco@

albertahealthservices.ca

\begin{abstract}
Advanced or metastatic gastric cancer constitutes the majority of patients in clinical practice. In North America, about $70 \%$ of cases are advanced or metastatic when diagnosed, which is higher than the $50 \%$ reported in Japan. This difference in presentation is reflected in 5 -year overall survival, which is about $20 \%$ in North America and $40 \%-60 \%$ in Japan. Despite numerous efforts of randomized studies on advanced gastric cancer, no globally accepted standard regimen has yet been established. Systemic chemotherapy provides palliation and prolongs survival, but the prognosis remains poor. Several monotherapies and combined regimens are currently available and vary around the world. Additionally, several molecular targeting agents are under evaluation in international randomized studies. Human epidermal growth factor receptor-2 (HER-2) is overexpressed or amplified in approximately $22 \%$ of patients with gastric cancer. Trastuzumab, a recombinant humanized anti-HER-2 monoclonal antibody, is the first biological therapy that has showed a survival improvement by nearly three months (reduced risk of death by $26 \%$ ). Therefore, trastuzumab in combination with cisplatin is a reasonable treatment option for patients with advanced gastric cancer who are HER-2 positive. This paper will focus on trastuzumab, its chemical and pharmacological characteristics, and the relevant efficacy, safety, and tolerability studies.
\end{abstract}

Keywords: gastric cancer, HER-2, trastuzumab

\section{Management issues in stomach cancer}

Gastric cancer is a cause of considerable morbidity and mortality the second leading cause of cancer-related death, and the third most common malignancy worldwide. ${ }^{1}$ In the US, it was estimated that there would be 21,000 new cases and 10,570 deaths from gastric cancer in 2010. ${ }^{1}$ In Japan, there are more than 100,000 new cases diagnosed and 50,000 who die annually. ${ }^{2}$ In Western countries, most newly diagnosed cases (73\%) will present in the advanced stages when gastric cancer is incurable, having a median survival of less than 1 year. Although $52 \%$ of gastric cancer cases in Japan are diagnosed at an early stage, this fact may be related to mass screening., ${ }^{3,4}$

Fluoropyrimidines, platinums, taxanes, and irinotecan are considered the main active cytotoxic agents for advanced gastric cancer. Palliative chemotherapy for advanced or metastatic gastric cancer offers significant advantages in selected fit patients, including increased survival, symptom control, and quality of life, compared with best supportive care alone. ${ }^{5-9}$ Systematic review showed that the overall survival benefit of chemotherapy over best supportive care was six months (hazards ratio [HR] 0.39, 95\% confidence interval [CI]: 0.28-0.52). A three-drug regimen including 5-flurouracil, anthracyclines, and cisplatin has showed the best survival results, 
with an HR of 0.83 (95\% CI: 0.74-0.93) over single-agent 5-flurouracil-based chemotherapy. ECF (epirubicin + cisplatin + infusional 5 -fluoruracil) response rates are $40 \%$, with a median survival of 9.4 months and $40 \%$ of patients alive at 1 year. ${ }^{10}$ When oxaliplatin is substituted for cisplatin, and capecitabine for 5-flurouracil (EOX), response rates are $48 \%$, median survival 11.2 months, and 1-year survival $48 \%$. Interestingly, thromboembolic events were significantly higher in cisplatin groups than in oxaliplatin groups (15.1 versus $7.6 \%, P<0.001) .{ }^{11}$

Docetaxel combined with cisplatin \pm 5-fluorouracil (DCF) has better response rates, longer progression-free survival, and a small survival advantage compared with cisplatin +5 -fluorouracil $(9.2$ versus 8.6 months, $P=0.02){ }^{12,13}$ However, in patients older than 65 years, increased toxicity of neutropenic infection and diarrhea has been seen. Irinotecan is also an active drug for advanced gastric cancer, although no improvement in survival has been demonstrated in randomized trials. ${ }^{14-16}$ It may be a more appropriate choice than cisplatin + 5-fluorouracil, given its better tolerance. DCF and 5-fluorouracil plus irinotecan regimens have not been directly compared against ECF.

Tegafur, a 5-fluorouracil prodrug, is mainly used in Japan. Phase III trials have demonstrated that tegafur is not inferior to 5-fluorouracil in overall survival, and it is associated with higher response rates, longer progression-free survival, longer time to treatment failure, and longer nonhospitalized survival. ${ }^{17}$ Tegafur combined with irinotecan was not significantly better compared with tegafur alone. ${ }^{18}$ However, when it was combined with cisplatin, significantly longer survival was seen than for tegafur alone, with acceptable toxicities. This regimen is standard therapy for metastatic gastric cancer in Japan. ${ }^{19}$

Although a large number of chemotherapy regimens have been proven in Phase III studies, there is no internationally accepted standard of care. Monotherapy with 5-fluorouracil or doublets with 5-fluorouracil and cisplatin, irinotecan, or an anthracycline, are reasonable options for patients who are not candidates for ECF. DCF may be recommended in very fit selected patients. Additionally, a preliminary updated meta-analysis suggested that chemotherapy combinations including irinotecan, oxaliplatin, docetaxel, or oral 5-fluouoracil prodrugs, are alternative treatment options to cisplatin/5-fluorouracil or cisplatin/5-fluorouracil/ anthracycline-combinations, but do not provide significant advantages in overall survival. ${ }^{20}$ Median time to progression after first-line chemotherapy for metastatic gastric cancer is typically $3-5$ months. No second-line regimen has been established, and historically few patients are considered for second-line treatment $(20 \%)$. However, recent data from a randomized trial showed that $39 \%-48 \%$ of patients in a sequence of chemotherapy including ECF followed by FOLFIRI (leucovorin + 5-fluorouracil + irinotecan) and the reverse sequence, received a second-line chemotherapy. ${ }^{21}$ So far, there are only preliminary data from a Phase III study in 40 patients that compared irinotecan monotherapy versus best supportive care in second line, demonstrating that irinotecan significantly prolongs overall survival (by 50.5 days) and improves tumor-related symptoms. ${ }^{22}$ Some Phase II studies has been published; taxanes and irinotecan are the most commonly used drugs in this setting, as monotherapy or in combined chemotherapy. Responses vary from $0 \%$ to $50 \%$, and time to progression and overall survival has been reported to be 3-6 and 6-9 months, respectively. Predictors of response have been described, including performance status, locally advanced rather than metastatic disease, and previous response to first-line therapy. ${ }^{23-26}$

Molecular targeting agents, alone or in combination with chemotherapy, are being tested in the second-line setting. ${ }^{27-29}$ Despite the benefits of palliative chemotherapy, and the diversity of chemotherapy regimens, the prognosis of advanced gastric cancer remains poor, with a median overall survival of 7-10 months. An increased understanding of molecular pathways has provided novel targets to treat cancer patients. Several molecularly targeted agents are under evaluation in patients with advanced gastric cancer. ${ }^{30,31}$

Bevacizumab combined with chemotherapy (cisplatin and irinotecan; oxaliplatin and docetaxel or 5-fluorouracil; DCF) has shown promising results in Phase II studies including treated and untreated patients (response rates $63 \%-71 \%$ ). However, the main concern arising from these trials is toxicity. ${ }^{32-35}$

A recent update of a Phase III trial including 774 patients with advanced gastric cancer who received combined chemotherapy (capecitabine/5-fluorouracil + cisplatin) with bevacizumab or placebo did not show significant differences in overall survival (12.1 versus 10.1 months, respectively), although there were significant improvements in progression-free survival and overall response rate, with an acceptable safety profile favoring the bevacizumab arm.

Sunitinib has demonstrated disease control rates of $40 \% .{ }^{36}$ Additionally, preliminary results showed encouraging response rates when sunitinib is combined with chemotherapy, such as tegafur, capecitabine + cisplatin, or capecitabine + oxaliplatin. ${ }^{37-39}$ Sorafenib in combination with docetaxel and 
cisplatin showed an encouraging efficacy profile, with tolerable toxicity ( $40 \%$ partial response rate). ${ }^{40}$

Cetuximab showed poor response rates $(5 \%)$ in pretreated patients. ${ }^{41}$ However, in combination with 5-fluorouracil and oxaliplatin or irinotecan, a relative risk of $52 \%-65 \%$ was seen in previously untreated patients. ${ }^{42-44}$ Cetuximab in combination with docetaxel and cisplatin has a response rate of $42 \%{ }^{45}$

Addition of matuzumab to EOX did not improve tumor response or survival in patients with advanced gastric cancer. ${ }^{46}$ Most clinical trials using epidermal growth factor receptor tyrosine kinase inhibitors in gastric cancer have shown minimal efficacy. Erlotinib had a response rate of $10 \%$ in previously untreated patients, ${ }^{47}$ and gefitinib had an $18 \%$ stable disease rate in previously treated patients. ${ }^{48}$ Preliminary results with lapatinib showed a $7 \%$ and $20 \%$ partial response and stable disease rate, respectively, in untreated patients. ${ }^{49}$ A preliminary report of a Phase II study showed that in combination with capecitabine, a partial response and stable disease were seen in $24 \%$ and $34 \%$, respectively. ${ }^{50}$ The EOX regimen with or without panitumumab (REAL-3 study) is ongoing, ${ }^{51}$ with Phase I trials of the combination showing a response rate of $65 \% .^{52}$

\section{Pharmacology and pharmacokinetics}

Human epidermal growth factor receptor (HER)-2/neu (c-erbB-2) is a transmembrane tyrosine kinase receptor, and a member of the HER family (HER-1, 2, 3, and 4). HER-2 functions as an oncogene. Gene amplification induces protein overexpression in cell membranes, and regulates signal transduction in cellular processes, including proliferation, differentiation, and cell survival. ${ }^{53,54}$ Aberrant HER-2 expression or function has been implicated in breast and gastric carcinogenesis, and is evident in other cancer types, including ovarian, salivary gland, and prostate and lung cancers..$^{53,55}$

Trastuzumab is a recombinant humanized anti-HER-2 MAb (rhuMAb HER-2), directed against the HER-2 extracellular domain. ${ }^{55}$ Several trastuzumab antitumor activities have been described ${ }^{53,56}$ (see Figure 1):

- Blocking of HER-2 receptor cleavage and inhibiting dimerization

- Increasing receptor destruction by endocytosis

- Inhibiting intracellular pathway signaling such as phosphoinositide 3-kinase signaling; may indirectly modulate proangiogenic and antiangiogenic factors, such as vascular endothelial growth factor production

- Inducing G1 phase arrest by inducing the cyclindependent kinase inhibitor, p27 Kip1
- Cytostatic and cytotoxic activity due to immune system recruitment by antibody-dependent cell-mediated cytotoxicity.

The pharmacokinetics and pharmacodynamics of trastuzumab are not completely understood. Most studies have been done in breast cancer patients. Trastuzumab has a low systemic clearance $(5.15 \pm 2.45 \mathrm{~mL} / \mathrm{kg} /$ day $)$ and a low volume of distribution $(44 \mathrm{~mL} / \mathrm{kg})$. The half-life may vary from 1.1 to 28 days with infusions of $10 \mathrm{mg}$ and $500 \mathrm{mg}$, respectively. Half-lives of 2.7, 3.1, 8.8, and 10.4 days were found after single doses of $1,2,4$, and $8 \mathrm{mg} / \mathrm{kg}$, respectively, suggesting that, after trastuzumab receptors become saturated, total body clearance becomes linear. Doses of $4 \mathrm{mg} / \mathrm{kg}$ and $8 \mathrm{mg} / \mathrm{kg}$ are enough to achieve and maintain the serum minimum concentrations $(10 \mu \mathrm{g} / \mathrm{mL})$ needed for antiproliferative effects and antibody-dependent cell-mediated cytotoxicity. ${ }^{57}$ Using a loading dose of $4 \mathrm{mg} / \mathrm{kg}$ followed by $2 \mathrm{mg} / \mathrm{kg} /$ week, trastuzumab achieves and maintains serum minimum concentrations greater than $20 \mu \mathrm{g} / \mathrm{mL}^{58}$

No drug interactions have been reported. No monoclonal antibody has been found to interact with major molecular pharmacokinetic determinants, such as enzymes, drug transporters, or orphan nuclear receptors. Using an initial dose of $4 \mathrm{mg} / \mathrm{kg}$ followed by $2 \mathrm{mg} / \mathrm{kg} /$ week, in combination with chemotherapy, the mean peak serum trastuzumab concentration is similar to that in patients receiving trastuzumab alone (101.0 $\mu \mathrm{g} / \mathrm{mL}$ at week 8 and $53.4 \mu \mathrm{g} / \mathrm{mL}$ of mean minimum concentration).$^{58,59}$ Elimination pathways are not yet defined, and the clinical relevance of trastuzumab kinetic variability is unknown. However, trastuzumab elimination seems to depend on high serum levels of circulating HER-2 extracellular domains, which can be cleaved from the surfaces of cancer cells by matrix metalloproteinase and released into the serum. Patients with high HER-2 extracellular domain levels tend to have a shorter trastuzumab half-life and lower minimum concentrations. Patients with four or more metastatic sites have faster clearance, independent of HER-2 extracellular domain levels. This is influenced little by trastuzumab exposure, and trastuzumab steady-state plasma levels are normally much higher than those of HER-2 extracellular domain levels. ${ }^{58,59}$

\section{Efficacy studies in gastric cancer}

Overexpression and amplification of HER-2/ErbB2 in gastric cancer vary widely, from $6 \%$ to $45 \%{ }^{59-64}$ The largest report screened 3883 patients with advanced gastric cancer from 24 countries, and found $22.1 \%$ HER-2-positivity rates in 3807 evaluated patients. Immunohistochemical and fluorescence in situ hybridization concordance was $87.3 \%$. HER-2 positivity 


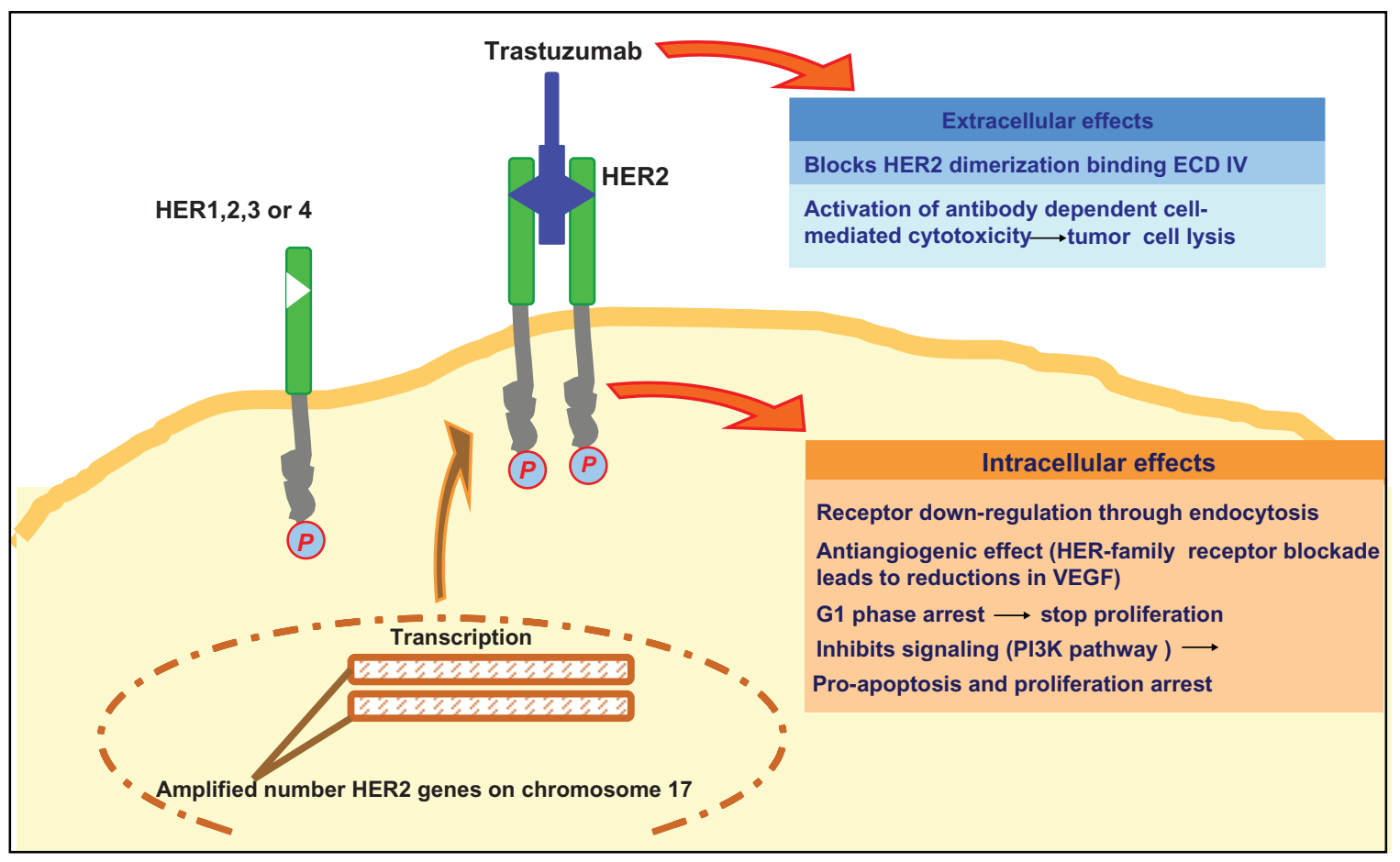

Figure I Antitumor effects of trastuzumab.

Abbreviations: ECD, extracellular domain; VEGF, vascular endothelial growth factor; PI3K, phosphoinositide 3-kinase.

was higher in gastroesophageal junction cancers than in gastric cancers $(33.2 \%$ versus $20.9 \%, P<0.001)$, and higher in intestinal cancers than in diffuse/mixed cancers $(32.2 \%$ versus $6.1 \% / 20.4 \%, P<0.001)$. No differences were seen between European and Asian countries. ${ }^{65}$

Early studies in HER-2-positive gastric cancer cell lines, showed the growth inhibitory effects of trastuzumab. Additionally, when combined with doxorubicin, cisplatin, or paclitaxel, it demonstrated increased cytotoxicity, suggesting that a regimen based on these combinations could be considered for gastric cancer ${ }^{66}$ (see Table 1).

Table I Trastuzumab in gastric cancer: Preclinical and clinical trials

\begin{tabular}{|c|c|c|c|}
\hline Authors & Type of study & $\mathbf{n}$ & Comments \\
\hline \multirow[t]{2}{*}{ Gong et al ${ }^{66}$} & Preclinical & Cell lines & Antitumor activity \\
\hline & $\mathrm{T}+\mathrm{D}$ & & \\
\hline \multirow[t]{2}{*}{ Cortés-Funes et al ${ }^{67}$} & Phase II & 21 & PR 35\% \\
\hline & $T+C$ & & SD $17 \%$ \\
\hline \multirow[t]{2}{*}{ Egamberdiev et $\mathrm{al}^{68}$} & Phase II & 16 & OR $55 \%$ \\
\hline & $\mathrm{T}+\mathrm{C}$ & & OS 8 months \\
\hline \multirow[t]{5}{*}{ Van Cutsem E et al ${ }^{69}$} & Phase III & 597 & OS $(P=0.0048$ \\
\hline & $\mathrm{T}+\mathrm{Cap} / 5-\mathrm{FU}$ & & death risk \\
\hline & $\mathrm{CT}$ alone & & reduction by $26 \%$ ) \\
\hline & & & 13.5 months \\
\hline & & & II.I months \\
\hline
\end{tabular}

Abbreviations: T, trastuzumab; C, cisplatin; D, doxorubicin; Cap, capecitabine; $\mathrm{CT}$, chemotherapy; PR, partial response; SD, stable disease; OS, overall survival; $\mathrm{OR}$, objective response; 5-FU, 5-fluorouracil.
Cortés-Funes et $\mathrm{al}^{67}$ have reported the preliminary results of a small Phase II study of 21 patients with advanced gastric cancer and HER-2 overexpression/amplification who received trastuzumab $8 \mathrm{mg} / \mathrm{kg}$ as a loading dose in the first cycle, followed by $6 \mathrm{mg} / \mathrm{kg}$ every 21 days, and cisplatin $75 \mathrm{mg} / \mathrm{m}^{2}$ every 21 days. These patients showed response rates of $35 \%$ and stable disease of $17 \%$. The therapy was well tolerated, with no Grade 4 toxicity reported.

Similarly, Egamberdiev et al ${ }^{68}$ in a small preliminary study of 16 patients with HER-2-positive advanced gastric cancer treated with trastuzumab $6 \mathrm{mg} / \mathrm{kg}$ and chemotherapy (cisplatin, 5-fluorouracil, and leucovorin) every three weeks, found an objective response rate of $55 \%$ and a median overall survival of eight months.

The ToGA study is the first randomized, controlled Phase III trial to evaluate the efficacy and safety of trastuzumab in advanced HER-2-positive gastric cancer. Five hundred and ninety-four patients received either trastuzumab in combination with 5-fluorouracil/capecitabine and cisplatin or chemotherapy alone. The chemotherapy group (control arm) received capecitabine $1000 \mathrm{mg} / \mathrm{m}^{2}$ twice a day for 14 days or continuous infusion of 5-fluorouracil $800 \mathrm{mg} / \mathrm{m}^{2} /$ day on days $1-5$ of each cycle, plus cisplatin $80 \mathrm{mg} / \mathrm{m}^{2}$ on day 1 every three weeks. The experimental arm received the same chemotherapy plus trastuzumab $8 \mathrm{mg} / \mathrm{kg}$ on day 1 of the first cycle, followed by $6 \mathrm{mg} / \mathrm{kg}$ every three weeks. The final report at 18.6 months of 
median follow-up for the experimental arm and 17.1 months for the control arm showed better median survival with the combination (13.8 versus 11.1 months, $P=0.0046$; HR $0.74,95 \%$ CI: $0.60-0.91)$ with a $26 \%$ reduction in risk of death. Progression-free survival was 6.7 versus 5.5 months $(P=0.0002)$, and tumor response (including complete and partial response) was $47 \%$ versus $35 \%(P=0.0017)$ with the addition of trastuzumab versus chemotherapy alone. Toxicity, including cardiac adverse events, was similar in both groups. More than $40 \%$ of patients in both groups received second-line treatment. Subgroup analysis for survival favored trastuzumab throughout (site of tumor, performance status, fluoropyrimidine used, histology, age, region, prior gastrectomy, and number of metastatic sites). Patients with high immunohistochemical positivity for HER-2 had a trend for better survival in the preplanned analysis (16 months) with trastuzumab compared with chemotherapy alone (11.8 months) ${ }^{69}$ This finding suggests that levels of HER2 protein expression predict response to trastuzumab, similar to what has been described in metastatic breast cancer. Therefore, patients with advanced gastric cancer and these tumor characteristics should be offered trastuzumab plus chemotherapy as a treatment option.

\section{Safety and tolerability}

The most common adverse events with trastuzumab are infusion-related reactions (fever, rigors, chills, nausea, dyspnea, and hypotension), present in about $40 \%$ of patients with the first dose and in 5\% with subsequent doses. ${ }^{70}$ Myelosuppression, nausea, and vomiting are rare, and alopecia has not been reported with monotherapy. However, quantifying the contribution of trastuzumab to these side effects is difficult when it is given in combination with chemotherapy. ${ }^{71}$ In the ToGA trial, serious adverse events were reported in $32 \%$ of patients treated with trastuzumab plus chemotherapy and $28 \%$ in the chemotherapy alone group. Treatment-related mortality was 3\% and $1 \%$ in the experimental and control arm, respectively.

Special attention has focused on the cardiotoxicity of trastuzumab. Sporadic cases of congestive heart failure were reported in the early trials. However, an association between impairment of left ventricular ejection fraction and trastuzumab was more evident when given in combination with chemotherapy. The pivotal study in breast cancer showed cardiac toxicity in $27 \%$ of patients when trastuzumab was combined with anthracyclines, in $13 \%$ when it was combined with paclitaxel, and in 5\% with trastuzumab alone. ${ }^{71}$ The ToGA trial reported cardiac event rates of $6 \%{ }^{70}$

Postmarketing surveillance for trastuzumab has reported that 62 of $25,000(0.002 \%)$ patients have had serious adverse events (hypersensitivity reactions, infusion-related reactions, and pulmonary events). Adult respiratory distress syndrome, anaphylaxis, and death within 24 hours of a trastuzumab infusion were reported. Fatal events occurred mostly in patients with pre-existing pulmonary dysfunction, so these patients should be treated with caution, and trastuzumab should be discontinued if severe infusion-related reactions occur. ${ }^{58,72} \mathrm{~A}$ Phase IV trial is currently underway to evaluate the efficacy and safety of trastuzumab in patients with advanced HER-2 gastric cancer.

\section{Patient-focused perspectives}

Ideally, any gain in survival should also be accompanied by an improvement in or at least stable quality of life. Assessment of quality of life with trastuzumab therapy has been studied in breast cancer patients, with favorable results. There are no available data in patients with gastric cancer as yet. Osoba et $\mathrm{al}^{73}$ found that patients treated with trastuzumab plus chemotherapy had significantly better improvement in quality of life (51\%) than patients treated with chemotherapy alone $(36 \%)$.

More recently, Rugo et $\mathrm{al}^{74}$ demonstrated that patients treated with chemotherapy and trastuzumab versus chemotherapy alone had more improvement in quality of life ( $51 \%$ versus $36 \%$, respectively). Improved physical, role functioning, and fatigue were also seen in the combined therapy arm.

\section{Discussion}

Gastric cancer is an aggressive disease with a high mortality rate, and despite recent progress in diagnosis, surgical techniques, chemotherapy, and radiotherapy, the prognosis remains poor. Advanced or metastatic gastric cancer constitutes the majority of patients in Western clinical practice. Chemotherapy has been considered the standard, with the significant advantages of increased survival, symptom control, and quality of life, compared with best supportive care alone. Trastuzumab is the first monoclonal antibody that has been shown to prolong life in patients with a malignant epithelial condition. In HER-2 breast cancer patients, its impact has been considerable. For advanced gastric cancer patients, trastuzumab is the first biological therapy that has showed a survival benefit. The ToGA trial included patients selected for treatment according to molecular profile. Encouragingly, trastuzumab in combination with chemotherapy showed a median survival of 13.8 months. Trastuzumab is a reasonable treatment option for patients with HER-2-positive advanced gastric cancer, although only approximately $20 \%$ of patients would be potential candidates. Some patients with 
HER-2-positive disease demonstrated primary or secondary resistance. Mechanisms of resistance and potential strategies to overcome these have been extensively researched in breast cancer, including loss of phosphatase and tensin homolog protein, activating mutations in the gene encoding phosphatidylinositol kinase-3, and increased signaling through other receptors (epithelial growth factor receptor and insulin-like growth factor-1 receptor). Lapatinib, the dual HER-2/epithelial growth factor receptor inhibitor, prolongs time to progression in patients with trastuzumab-resistant HER-2-positive breast cancer, ${ }^{75,76}$ and warrants evaluation in trastuzumab-resistant HER-2-positive gastric cancer.

Two Phase II trials, one combining chemotherapy (capecitabine and oxaliplatin) and targeted therapy (bevacizumab and trastuzumab), and the other with trastuzumab in combination with tegafur and cisplatin in advanced gastric cancer are planned. Additionally, two Phase II studies evaluating lapatinib in combination with capecitabine and weekly paclitaxel in first-line and second-line settings, respectively, in advanced gastric cancer are open to recruitment.

Further research is needed to evaluate the efficacy of trastuzumab as monotherapy, maintenance treatment, and second-line therapy for advanced gastric cancer. Moreover, trastuzumab should be integrated into curative treatment trials for patients with gastric cancer, such as perioperative or postoperative therapy.

With a better understanding of gastric cancer epidemiology and an ability to categorize it into distinct clinical and pathologic entities, improvement in gastric cancer therapy is expected. Moreover, by improving our knowledge of gastric cancer biology and signaling pathways, integration of targeted therapies has become possible and promising. Better selection of patients for a particular therapy will significantly improve treatment paradigms for this deadly disease, with the possibility of also improving patient survival. Several clinical trials with targeted therapies, including bevacizumab, cetuximab, panitumumab, and lapatinib in combination with conventional chemotherapy regimens, are currently ongoing, which may help to increase our armamentarium against gastric cancer.

\section{Disclosure}

The authors report no conflicts of interest in this work.

\section{References}

1. American Cancer Society. Cancer Facts and Figures 2010. Atlanta, GA: American Cancer Society; 2010.

2. Sugano K. Gastric cancer: Pathogenesis, screening, and treatment. Gastrointest Endosc Clin N Am. 2008;18(3):513-522.
3. Inoue M, Tsugane S. Epidemiology of gastric cancer in Japan. Postgrad Med J. 2005;81(957):419-424.

4. Foukakis T, Lundell L, Gubanski M, et al. Advances in the treatment of patients with gastric adenocarcinoma. Acta Oncol. 2007;46(3):277-285.

5. Murad AM, Santiago FF, Petroianu A, et al. Modified therapy with 5-fluorouracil, doxorubicin, and methotrexate in advanced gastric cancer. Cancer. 1993;72(1):37-41.

6. Pyrhönen S, Kuitunen T, Nyandoto P, et al. Randomised comparison of fluorouracil, epidoxorubicin and methotrexate (FEMTX) plus supportive care with supportive care alone in patients with non-resectable gastric cancer. Br J Cancer. 1995;71(1):587-591.

7. Glimelius B, Ekstrom K, Hoffman K, et al. Randomized comparison between chemotherapy plus best supportive care with best supportive care in advanced gastric cancer. Ann Oncol. 1997;8(2):163-168.

8. Janunger KG, Hafström L, Glimelius B. Chemotherapy in gastric cancer: A review and updated meta-analysis. Eur J Surg. 2002;168(11):597-608.

9. Wagner AD, Grothe W, Haerting J, et al. Chemotherapy in advanced gastric cancer: A systematic review and meta-analysis based on aggregate data. J Clin Oncol. 2006;24(18):2903-2909.

10. Ross P, Nicolson M, Cunningham D, et al. Prospective randomized trial comparing mitomycin, cisplatin, and protracted venous-infusion fluorouracil (PVI 5-FU) with epirubicin, cisplatin, and PVI 5-FU in advanced esophagogastric cancer. J Clin Oncol. 2002;20(8):1996-2004.

11. Cunningham D, Starling N, Rao S, et al; Upper Gastrointestinal Clinical Studies Group of the National Cancer Research Institute of the United Kingdom. Capecitabine and oxaliplatin for advanced esophagogastric cancer. N Engl J Med. 2008;358(1):36-46.

12. Van Cutsem E, Moiseyenko VM, Tjulandin S, et al. Phase III study of docetaxel and cisplatin plus fluorouracil compared with cisplatin and fluorouracil as first-line therapy for advanced gastric cancer: A Report of the V325 Study Group. J Clin Oncol. 2006;24(31):4991-4997.

13. Ajani JA, Moiseyenko VM, Tjulandin S, et al. Clinical benefit with docetaxel plus fluorouracil and cisplatin compared with cisplatin and fluorouracil in a phase III trial of advanced gastric or gastroesophageal cancer adenocarcinoma: The V-325 Study Group. J Clin Oncol. 2007;25(22):3205-3209.

14. Bouche O, Raoul JL, Bonnetain F, et al. Randomized multicenter phase II trial of a biweekly regimen of fluorouracil and leucovorin (LV5FU2), LV5FU2 plus cisplatin, or LV5FU2 plus irinotecan in patients with previously untreated metastatic gastric cancer: A Federation Francophone de Cancerologie Digestive Group Study - FFCD 9803. J Clin Oncol. 2004;22(21):4319-4328.

15. Moehler M, Eimermacher A, Siebler J, et al. Randomized phase II evaluation of irinotecan plus high-dose 5-fluorouracil and leucovorin (ILF) versus 5-fluorouracil, leucovorin, and etoposide (ELF) in untreated metastatic gastric cancer. Br J Cancer. 2005;92(12):2122-2128.

16. Dank M, Zaluski J, Valvere V, et al. Randomized phase III trial of irinotecan (CPT 11) 5-FU/folinic acid (FA) vs CDDP-5-FU in first line advanced gastric cancer patients. J Clin Oncol. 2005;23 Suppl 16: S4003.

17. Boku N, Yamamoto S, Shirao K, et al. Randomized phase III study of 5-fluorouracil (5-FU) alone versus combination of irinotecan and cisplatin (CP) versus S-1 alone in advanced gastric cancer (JCOG9912). J Clin Oncol. 2007;25 Suppl 18:SLBA4513T.

18. Imamura $H$, IIishi $H$, Tsuburaya A, et al. Randomized phase III study of irinotecan plus S-1 (IRIS) versus S-1 alone as first-line treatment for advanced gastric cancer (GC0301/TOP-002). Poster A5 presented at the ASCO Gastrointestinal Cancers Symposium, San Francisco, CA, January 15-17, 2008 .

19. Koizumi W, Narahara H, Hara T, et al. S-1 plus cisplatin versus S-1 alone for first-line treatment of advanced gastric cancer (SPIRITS trial): A phase III trial. Lancet Oncol. 2008;9(3):215-221.

20. Wagner AD, Unerzagt S, Grothe W, et al. Novel chemotherapy combinations in advanced gastric cancer: An updated meta-analysis. Ann Oncol. 2010;21 Suppl 8:S740. 
21. Guimbaud R, Louvet C, Bonnetain F, et al. Final results of the intergroup FFCD-GERCOR-FNCLCC 03-07 phase III study comparing two sequences of chemotherapy in advanced gastric cancers. Ann Oncol. 2010;21 Suppl 8:S8010.

22. Thuss-Patience PC, Kretzschmar A, Deist T, et al. Irinotecan versus best supportive care (BSC) as second-line therapy in gastric cancer: A randomized phase III study of the Arbeitsgemeinschaft Internistische Onkologie (AIO). J Clin Oncol. 2009;27 Suppl 5:S4540.

23. Shimoyama R, Yasui H, Boku N, et al. Weekly paclitaxel for heavily treated advanced or recurrent gastric cancer refractory to fluorouracil, irinotecan, and cisplatin. Gastric Cancer. 2009;12(4):206-211.

24. Takahari D, Shimada Y, Takeshita S, et al. Second-line chemotherapy with irinotecan plus cisplatin after the failure of S-1 monotherapy for advanced gastric cancer. Gastric Cancer. 2010;13(3):186-190.

25. Wilson D, Hiller L, Geh JI. Review of second-line chemotherapy for advanced gastric adenocarcinoma. Clin Oncol (R Coll Radiol). 2005;17(2):81-90.

26. Boku N. Chemotherapy for metastatic gastric cancer in Japan. Int $J$ Clin Oncol. 2008;13(6):483-487.

27. Bang YJ, Kang YK, Kang WK, et al. Phase II study of sunitinib as second-line treatment for advanced gastric cancer. Invest New Drugs. 2010 May 12. [Epub ahead of print].

28. Yoon DH, Ryu1 M, Lee J, et al. Phase II study of everolimus in patients with advanced gastric cancer refractory to chemotherapy including fluoropyrimidine and platinum. Ann Oncol. 2010;21 Suppl 8:S725.

29. Tebbutt NC, Sourjina T, Strickland AH, et al. ATTAX2: Docetaxel plus cetuximab as second-line treatment for docetaxel refractory oesophago-gastric cancer - final results of a multicentre phase II trial by the AGITG. J Clin Oncol. 2008;26 Suppl 20:S15554.

30. Arkenau HT. Gastric cancer in the era of molecularly targeted agents: current drug development strategies. J Cancer Res Clin Oncol. 2009;135(2):855-866.

31. Ohtsu A. Chemotherapy for metastatic gastric cancer: Past, present, and future. J Gastroenterol. 2008;43(4):256-264.

32. Shah MA, Ramanathan RK, Ilson DH, et al. Multicenter phase II study of irinotecan, cisplatin and bevacizumab in patients with metastatic gastric or gastroesophageal junction adenocarcinoma. J Clin Oncol. 2006;24(33):5201-5206.

33. Enzinger PC, Fidias P, Meyerhardt J, et al. Phase II study of bevacizumab and docetaxel in metastatic esophageal and gastric cancer. Poster A68 presented at the ASCO Gastrointestinal Cancers Symposium, San Francisco, CA, January 26-28, 2006.

34. Cohenuram MK, Lacy J. FOLFOX6 and bevacizumab (FOLFOX6/B) for metastatic esophageal (E), gastroesophageal (GE), and gastric (G) adenocarcinoma: A single institution's initial clinical experience. Poster A74 presented at the ASCO Gastrointestinal Cancers Symposium, San Francisco, CA, January 15-17, 2008.

35. Hammad N, Philip PA, Shields AF, et al. A phase II study of bevacizumab, docetaxel, and oxaliplatin in gastric and gastroesophageal junction (GEJ) cancer. Poster A30 presented at the ASCO Gastrointestinal Cancers Symposium, San Francisco, CA, January 15-17, 2008.

36. Bang YJ, Kang Y, Kang W, et al. Sunitinib as second line treatment for advanced gastric cancer: Preliminary results from a phase II study. J Clin Oncol. 2007;25 Suppl 18:S4603.

37. Park SR, Lee K, Oh D, et al. Sunitinib (su) with cisplatin (p) and capecitabine (x) or oxaliplatin (o) and $\mathrm{x}$ (xelox) in advanced gastric cancer (gc)-a phase I, dose-finding study. Ann Oncol. 2010;21 Suppl 8: S815.

38. Muro K, Miyata Y, Li M, Watanabe K, Boku N. Cisplatin (P) in patients (pts) with advanced or metastatic gastric cancer (CG). Ann Oncol. 2010;21 Suppl 8:S816.

39. Gomez-Martın C, Gil-Martın M, Montagut C. A phase I, dose-finding study of sunitinib (su) in combination with cisplatin (p) and 5-fluorouracil (5-fu) in patients (pts) with advanced gastric cancer ( $\mathrm{gc}$ ). Ann Oncol. 2010;21 Suppl 8:S818.
40. Sun W, Powell ME, O'Dwyer P, et al. Phase II study of sorafenib in combination with docetaxel and cisplatin in the treatment of metastatic or advanced gastric and gastroesophageal junction adenocarcinoma: ECOG 5203. J Clin Oncol. 2010:28(18):2947-2951.

41. Stein A, Al-Batran SE, Arnold D, et al. Cetuximab with irinotecan as salvage therapy in heavily pretreated patients with metastatic gastric cancer. Poster A47 presented at the ASCO Gastrointestinal Cancers Symposium, San Francisco, CA, January 15-17, 2008.

42. Pinto C, Di Fabio F, Siena S, et al. Phase II study of cetuximab in combination with FOLFIRI in patients with untreated advanced gastric or gastroesophageal junction adenocarcinoma (FOLCETUX study). Ann Oncol. 2007;18(3):510-517.

43. Lordick F, Luber B, Lorenzen S, et al. Cetuximab plus oxaliplatin/ leucovorin/5-fluorouracil in first-line metastatic gastric cancer: A phase II study of the Arbeitsgemeinschaft Internistische Onkologie (AIO). Br J Cancer. 2010;102(3):500-505.

44. Kim C, Lee JL, Ryu MH, et al. A prospective phase II study of cetuximab in combination with XELOX (capecitabine and oxaliplatin) in patients with metastatic and/or recurrent advanced gastric cancer. Invest New Drugs. 2009 December 9. [Epub ahead of print].

45. Pinto C, Di Fabio F, Barone C, et al. Phase II study of cetuximab in combination with cisplatin and docetaxel in patients with untreated advanced gastric or gastro-oesophageal junction adenocarcinoma (DOCETUX study). Br J Cancer. 2009;101(8):1261-1268.

46. Rao S, Starling N, Cunningham D, et al. Matuzumab plus epirubicin, cisplatin and capecitabine (ECX) compared with epirubicin, cisplatin and capecitabine alone as first-line treatment in patients with advanced oesophago-gastric cancer: A randomised, multicentre open-label phase II study. Ann Oncol. 2010;21(11):2213-2219.

47. Dragovich T, McCoy S, Fenoglio-Preiser C, et al. SWOG 0127 phase II trial of erlotinib in GEJ and gastric adenocarcinoma. $J$ Clin Oncol. 2006;24(30):4922-4927.

48. Doi T, Koizumi W, Siena S, et al. Efficacy, tolerability and pharmacokinetics of gefitinib (ZD1839) in pretreated patients with metastatic gastric cancer. Proc Am Soc Clin Oncol. 2003;22:Abstr 1036.

49. Iqbal S, Goldman B, Lenz HJ, et al. A phase II SWOG study of GW572016 (lapatinib) as first line therapy in patients (pts) with advanced or metastatic gastric cancer. J Clin Oncol. 2007;25 Suppl 8: S4621

50. Lenz H, Zhang W, Kemner AM. Lapatinib 1 capecitabine in advanced gastric cancer: An open-label phase II study of non ERBB2-targeted disease. Ann Oncol. 2010;21 Suppl 8:S817.

51. Okines AF, Ashley SE, Cunningham D, et al. Epirubicin, oxaliplatin, and capecitabine with or without panitumumab for advanced esophagogastric cancer: Dose-finding study for the prospective multicenter, randomized, phase II/III REAL-3 trial. $J$ Clin Oncol. 2010;28(25):3945-3950.

52. Rao S, Starling N, Cunningham D, et al. Phase I study of epirubicin, cisplatin and capecitabine plus matuzumab in previously untreated patients with advanced oesophagogastric cancer. Br J Cancer. 2008;99(6):868-874.

53. Baselga J, Swain SM. Novel anticancer targets: Revisiting ERBB2 and discovering ERB3. Nat Rev Cancer. 2009;9(7):463-475.

54. Halla PS, Cameron DA. Current perspective - trastuzumab. Eur $J$ Cancer. 2009;45(1):12-18.

55. Carter P, Presta L, Gorman CM, et al. Humanization of an antip185HER2 antibody for human cancer therapy. Proc Natl Acad Sci US A. 1992;89(10):4285-4289.

56. Valabrega G, Montemurro F, Aglietta M. Trastuzumab: Mechanism of action, resistance and future perspectives in HER2-overexpressing breast cancer. Ann Oncol. 2007;18(6):977-984.

57. Treish I, Schwartz R, Lindley C. Pharmacology and therapeutic use of trastuzumab in breast cancer. Am J Health Syst Pharm. 2000;57(22):2063-2076.

58. Bruno R, Washington CB, Lu JF, et al. Population pharmacokinetics of trastuzumab in patients with HER2+ metastatic breast cancer. Cancer Chemother Pharmacol. 2005;56(4):361-369. 
59. Marx AH, Tharun L, Muth J, et al. HER-2 amplification is highly homogenous in gastric cancer. Hum Pathol. 2009;40(6):769-777.

60. Barros-Silva JD, Leitão D, Afonso L, et al. Association of ERBB2 gene status with histopathological parameters and disease-specific survival in gastric carcinoma patients. Br J Cancer. 2009;100(3):487-493.

61. Kim MA, Jung EJ, Lee HS, et al. Evaluation of HER-2 gene status in gastric carcinoma using immunohistochemistry, fluorescence in situ hybridization, and real-time quantitative polymerase chain reaction. Hum Pathol. 2007;38(9):1386-1393.

62. Tanner M, Hollmen M, Junttila TT, et al. Amplification of HER-2 in gastric carcinoma: Association with topoisomerase II alpha gene amplification, intestinal type, poor prognosis and sensitivity to trastuzumab. Ann Oncol. 2005:16(2):273-278.

63. Yano T, Doi T, Ohtsu A, et al. Comparison of HER2 gene amplification assessed by fluorescence in situ hybridization and HER2 protein expression assessed by immunohistochemistry in gastric cancer. Oncol Rep. 2006;15(1):65-71.

64. Yu GZ, Chen Y, Wang JJ. Overexpression of Grb2/HER2 signaling in Chinese gastric cancer: their relationship with clinicopathological parameters and prognostic significance. J Cancer Res Clin Oncol. 2009;135(10):1331-1339.

65. Bang Y, Chung H, Xu J, et al. Pathological features of advanced gastric cancer (GC): Relationship to human epidermal growth factor receptor 2 (HER2) positivity in the global screening programme of the ToGA trial. J Clin Oncol. 2009;27 Suppl 15:S4556.

66. Gong SJ, Jin CJ, Rha SY, et al. Growth inhibitory effects of trastuzumab and chemotherapeutic drugs in gastric cancer cell lines. Cancer Lett. 2004;214(2):215-224.

67. Cortés-Funes H, Rivera F, Alés I, et al. Phase II of trastuzumab and cisplatin in patients (pts) with advanced gastric cancer (AGC) with HER2/ neu overexpression/amplification. J Clin Oncol. 2007;25 Suppl 18: S4613.
68. Egamberdiev DM, Djuraev MD, Tuydjanova K, Nematov ON. Our experience in the use of trastuzumab in patients with advanced stomach cancer. Ann Oncol. 2010;21 Suppl 8:S839.

69. Van Cutsem E, Kang Y, Chung H, et al; ToGA Trial Investigators. Trastuzumab in combination with chemotherapy versus chemotherapy alone for treatment of HER2-positive advanced gastric or gastro-oesophageal junction cancer (ToGA): A phase 3, open-label, randomized controlled trial. Lancet. 2010;376(9742):687-697.

70. Hudis CA. Trastuzumab - mechanism of action and use in clinical practice. $N$ Engl J Med. 2007;357(1):39-51.

71. Seidman A, Hudis C, Pierri MK, et al. Cardiac dysfunction in the trastuzumab clinical trials experience. J Clin Oncol. 2002;20(5):1215-1221.

72. González V, Salgueiro E, Jimeno FJ, et al. Post-marketing safety of antineoplasic MAb: Rituximab and trastuzumab. Pharmacoepidemiol Drug Saf. 2008;17(7):714-721.

73. Osoba D, Slamon DJ, Burchmore M, Murphy M. Effects on quality of life of combined trastuzumab and chemotherapy in women with metastatic breast cancer. J Clin Oncol. 2002;20(14): 3106-3113.

74. Rugo H, Brammer M, Zhang F, Lalla D. Effect of trastuzumab on health-related quality of life in patients with HER2-positive metastatic breast cancer: Data from three clinical trials. Clin Breast Cancer. 2010;10(4):288-293.

75. Cameron D, Casey M, Press M, et al. A phase III randomized comparison of lapatinib plus capecitabine versus capecitabine alone in women with advanced breast cancer that has progressed on trastuzumab: Updated efficacy and biomarker analyses. Breast Cancer Res Treat. 2008;112(3):533-543.

76. Geyer CE, Forster J, Lindquist D, et al. Lapatinib plus capecitabine for HER2-positive advanced breast cancer. New Engl J Med. 2006;355(26):2733-2743
Cancer Management and Research

\section{Publish your work in this journal}

Cancer Management and Research is an international, peer-reviewed open access journal focusing on cancer research and the optimal use of preventative and integrated treatment interventions to achieve improved outcomes, enhanced survival and quality of life for the cancer patient. The journal welcomes original research, clinical \& epidemiological

\section{Dovepress}

studies, reviews \& evaluations, guidelines, expert opinion \& commentary, case reports \& extended reports. The manuscript management system is completely online and includes a very quick and fair peerreview system, which is all easy to use. Visit http://www.dovepress.com/ testimonials.php to read real quotes from published authors. 